Gynäk. Rdsch. 1983;23:I-VI

\title{
Contents, Vol. 23, 1983
}

\section{No. 1 Originalarbeiten}

Enzymatische Aktivität in der embryo-fetalen Leber

Hrgovic, Z.; Schütte, H 1

Perinatale Morbidität und Mortalität bei der Beckenendlage. Gegenüberstellung der Ergebnisse der Städtischen Frauenklinik Braunschweig und der Universitäts-Frauenklinik

Zagreb

Hrgovic, Z.; Langer, H.; Barsic, E 11

Familiengründung, Kinderwunsch und Geburtenregelung bei Schweizer Ehepaaren - Ergebnisse einer repräsentativen Umfrage. I. Familiengründung und Kinderwunsch

Höpflinger, F.; Kühne, F 25

Kurzfassungen von Zeitschriftenartikeln

Die kombinierte Anwendung von Zytologie und Kolposkopie zur Erhöhung der diagno-

stischen Treffsicherheit bei präklinischen Läsionen der Cervix uteri

Lozowski, M.S.; Mishriki, Y.; Talebian, F.; Solitare, G 35

Verminderung des Wehenschmerzes durch transkutane elektrische Nervenstimulation:

Sicherheitsaspekte

Bundsen, P.; Ericson, K

Urodynamische Untersuchungen im normalen Menstruationszyklus: das Verhältnis

zwischen Hormonveränderungen im Verlaufe des Menstruationszyklus und Urethra-

druckprofil

Van Geelen, J.M.; Doesburg, W.H.; Thomas, C.M.G.; Martin, C.B., Jr

Elektrische Aktivität des menschlichen Eileiters während des Zyklus

Talo, A.; Pulkkinen, M.0 39

Rauchen während der Schwangerschaft: eine Ursache fur fetale Hypoxie - ein experimenteller Nachweis

Socol, M.L.; Manning, F.A.; Murata, Y.; Druzin, M.L

Orale Steroidkontrazeption: Vorteile für die Gesundheit

Mishell, D.R., Jr

43

Kadmiumspiegel im mütterlichen Blut, Nabelschnurblut und Plazentagewebe von schwangeren Raucherinnen

Kuhnert, P.M.; Kuhnert, B.R.; Bottoms, S.F.; Erhard, P

Ein Index zur Erfassung von Risikoschwangerschaften

Fortney, J.; Whitehorne, E.W 47

Die Uberlebensrate beim Endometriumkarzinom: Ein Vergleich von Frauen mit und ohne vorangegangene postmenopausale Östrogentherapie

Chu, J.; Schweid, A.I.; Weiss, N.S 48

Antenatale sonographische Diagnose einer Nabelschnurverletzung

Romero, R.; Chervenak, F.A.; Coustan, D.; Berkowitz, R.L.; Hobbins, J.C 50

Reining der Hypothalamus-Hypophysen-Eierstock-Achse bei adoleszenten Mädchen 
Lemarchand-Béraud, T.; Zufferey, M.-M.; Reymond, M.; Rey, 151

Chemosensibilitätstestung des Ovarial- und Mammakarzinoms - Möglichkeiten und

Grenzen verschiedener Methoden und ihre klinische Anwendung

Kaufmann, M.; Volm, M.; Mattern, J.; Kubli, F 53

Zur Frage der Rezidivinfekte nach Behandlung einer Trichomoniasis vaginalis mit Solco-

trichovac $\mathbb{R}$

Litschgi, M 55

Fortbildung

3. Winterfortbildungstagung der Österreichischen Gesellschaft für Gynäkologie und

Geburtshilfe. Obergurgl, 7.-13. Februar 1982 Überwachung von Toxoplasmose während der

Schwangerschaft

Aspöck, $\mathrm{H} \quad 57$

Tumormarker in der gynäkologischen Onkologie

Pickel, $\mathrm{H} \quad 66$

Inhaltsverzeichnis

III

Physiologische Bedeutung von Prostaglandinen fur den Geburtsmechanismus beim

Menschen

Husslein, $\mathrm{P} \quad 68$

Diagnosen von Atemstörungen des Neugeborenen im Kreisssaal. Die wichtigsten akuten

Atemstörungen des Neugeborenen unter Ausschluss von Asphyxie, Geburtstrauma und

Atemnotsyndrom

Rosegger, $\mathrm{H} \quad 70$

Kindliche Sexualität

Springer-Kremser, M 74

No. 2 Originalarbeiten

Familiengründung, Kinderwunsch und Geburtenregelung bei Schweizer Ehepaaren - Ergebnisse einer repräsentativen Umfrage. II. Kontrazeption in der Schweiz - Kenntnisse und

Praxis der Schweizer Ehepaare

Kühne, F.; Höpflinger, F 77

Hystérosalpingographie par sonde de Foley

Samim, F.; Habashi, V.; Hilan, W 88

Steht die Makrosomie der Neugeborenen bei Diabetes mellitus auch heute noch im Vordergrund?

Fischl, F.; Binstorfer, E.; Reinold, E 93

Postpartale Uterusinvolution und Laktationsmengen im randomisierten Vergleich von Pro-

$\operatorname{stin}^{3 / 4}{ }^{\circledR}$-Tabletten mit Methergin ${ }^{\circledR}$-Dragees

Grünberger, W 100

Sexualité, contraception et grossesse chez Tadolescente

Rey-Stocker, 1108

Kurzfassungen von Zeitschriftenartikeln

Eine Lebenszeitanalyse-Studie von Schwangerschaften ausgelöst durch niedrige standardisierte Dosen von Menotropin bei Patientinnen, die früher durch eine Clomiphen-ZitratTherapie anovulatorisch geblieben waren

Goldfarb, A.F; Schlaff, S.; Mansi, M.L

Ovarialkarzinom: Stellenwert der Douglaspunktion und CEA-Titer in der Peritonealflüs- 
sigkeit

Zylberberg, B.; Salat-Baroux, J.; Ravina, J.H.; Demarcourt, V.; Dormont, D

Prostaglandinsynthese von persistierendem proliferativem Endometrium

Smith, S.K.; Abel, M.H.; Kelly, R.W.; Baird, D.T 125

Vaginal-Spermizide - haben sie irgendeinen Einfluss auf eine eintretende Schwangerschaft?

Ergebnisse einer multizentrischen Studie

Huggins, G.; Vessey, M.; Flavel, R.; Yeates, D.; McPherson, K . . 127

Hypophysen- und Ovarialfunktion während der Kontrazeption mit dem subkutan implan-

tierten Progesteronderivat ST-1435

Lähteenmäki, Pertti; Weiner, E.; Lähteenmäki, Pekka; Johansson, E.D.B.; Luukkainen, T. 129

Die fibrinolytische Aktivität der Uterinflüssigkeit bei Frauen, die die Pille benützen

Casslen, B 130

Künstliche Scheide mittels Lyodura (Dura mater cerebri)

Beller, F.K.; Wagner, H 132

Prognostische Aussagekraft des Stadiums bei Patientinnen mit operiertem Endometriumkarzinom

Baltzer, J.; Lohe, K.J.; Kürzl, R.; Scheer, K.P.; Zander, J 134

Mögliche prognostische Kriterien zur Therapieverbesserung beim Mammakarzinom

Kaufmann, M.; Klinga, K 135

Zur Überprüfung der aktuellen uteroplazentaren Durchblutung mit der Plazentadurchströmungsmessung bei normalen Schwangerschaften

Voigt, R.; Stoll, W.; Hempel, E 137

IV

Inhaltsverzeichnis

Fortbildung

Nolvadex ${ }^{\circledR}$ (Tamoxifen) beim Endometriumkarzinom

Internationaler Workshop am 25. 5. 1982 in Macclesfield Einleitung

Gitsch, E 139

Auszug aus dem Einleitungs- und Spezialreferat

Taylor, R.W 140

Auszug aus dem Spezialreferat

Bonte, J 142

No. 3 Originalarbeiten

Der Einfluss mütterlicher Hyperventilation auf das fetale Kardiotokogramm

Huber, J.C; Müller, G.; Reinold, E 145

Rhesusprophylaxe und Verlauf nach Amniozentese in der Frühschwangerschaft

Herrmann, U.; Sidiropoulos, D 153

Untersuchungen zur ultrasonographischen Feststellung der Ovulation

Borruto, F.; Albrich, W 160

Zur Kiassifikation der Stressinkontinenz nach Ingelman-Sundberg

Schüssler, B.; Alloussi, S 166

Sirénomelie chez un fæus mort in utero, découverte par Гélévation de $\Gamma \alpha$-fætoprotéine

sérique maternelle

Marguerat, P.; Pescia, G.; Nguyen-The, H 175

Aktueller Stand der radiologischen Mammadiagnostik

Hohenberg, G.; Wolf, G 180 
Dauer der Schutzwirkung gegen Trichomoniasis nach Impfung mit SolcoTrichovac $₫$ Grcic, R.; Milovanovic, R.; Stavric, V 191

Kurzfassungen von Zeitschriftenartikeln

Auswirkungen der radikalen Hysterektomie und pelvinen Lymphonodektomie auf den unteren Harntrakt

Asmussen, M.; Ulmsten, U 197

Hormonelle und histologische Untersuchung der Lutealphase bei Frauen nach Aspiration des präovulatorischen Follikels

Frydman, R.; Testart, J.; Giacomini, P.; Imbert, M.C.; Martin, E.; Nahoul, K

Diagnose der intrauterinen Wachstumsretardierung - Vergleich von Klinik, Gesamtöstrogenbestimmung aus dem 24-h-Urin und Ultraschallbiometrie (Distanzmessungen, biparietaler Kopfdurchmesser und thorako-abdominaler Querdurchmesser) unter Berücksichtigung des antepartalen und subpartalen CTGs

Schmidt, W.; Kübli, F.; Garoff, L.; HendПk, H.J.; Leucht, W.; Runnebaum, B 200

Bericht über 5 Jahre Erfahrung mit intrauteriner Spülzytologie

Favre, J.; Bernard, P.; Besançon, D.; Siebert, S 202

Zur Frage der optimalen Therapie bei prämalignen Veränderungen der Cervix uteri

Singer, A.; Walker, P 203

Vorzeitige Follikelruptur bei mehrmaliger Ultraschalluntersuchung zum Ovulationszeitpunkt

Testart, J.; Thebault, A.; Souderes, E.; Frydman, R 205

Zytoplasmatische Steroidrezeptoren bei Ovarialkarzinomen

Quinn, M.A.; Pearce, P.; Rome, R.; Funder, J.W.; Fortune, D.; Pepperell, R.J 206

Die Hämokonzentration in der schweren Gestose

Sagen, N.; Koller, O.; Haram, K 208

Die Auswirkung des tiefen Plazentasitzes auf den mütterlichen Blutdruck und auf die

Plazentafunktion

Nicolaides, K.H.; Faratian, B.; Symonds, E.M 209

Behandlung des Zervixkarzinoms mit Bromocriptin

Guthrie, D 211

Inhaltsverzeichnis

$\mathrm{V}$

Vulvakarzinom: Analyse von Behandlungsfehlern

Podratz, K.C.; Symmonds, R.E.; Taylor, W.F 212

Die Behandlung des vorzeitigen Blasensprunges: ein Vergleich zwischen aktivem und konservativem Vorgehen

Barrett, J.M.; Boehm, F.H 214

Fortbildung

12. Fortbildungskurs der II. Universitäts-Frauenklinik Wien fur Fachärzte der Gynäkologie und Geburtshilfe. Obergurgl, 7.-13. Februar 1983 Vorwort

Gitsch, E 216

Das Lymphödem: Wie soil das Lymphödem nicht behandelt werden? Zur Frage der operativen Lymphödembehandlung

Földi, M 216

Neue Aspekte in der Therapie der schwangerschaftsinduzierten Hypertonie

Hammerle, A.F 
Therapie der schweren EPH-Gestose mit Prostazyklin

Dadak, Ch.; Sinzinger, H 225

Isotopen in Geburtshilfe und Gynäkologie aus der Sicht des Strahlenschutzes

Janisch, H 229

No. 4 Originalarbeiten

Systemtherapie vulvovaginaler Mykosen

Herzog, R.E 233

Klinische und histologische Resultate bei wiederholt fraglichen zytologischen Befunden von der Cervix uteri

Gitsch, E.; Ulm, R 242

Zur Frage des optimalen Operationsmodus des Korpuskarzinoms

Gitsch, E.; Kofler, E.; Kupka, St 250

Kurzfassungen von Zeitschriftenartikeln

Der Einfluss des Stillens auf die Geburtsfrequenz

Howie, P.W.; McNeilly, A.S 270

Der inhibitorische Wirkungsmechanismus des Prolaktins auf die ovarielle Funktion

McNeilly, A.S.; Glasier, A.; Jonassen, J.; Howie, P.W 271

Metronidazolprophylaxe bei der abdominalen Hysterektomie

Manthorpe, T.; Justesen, T 273

Fetale Gewichtsschätzung durch Ultraschallbiometrie und Berechnung nach einer neuen

Formel

Eik-Nes, S.H.; Grottum, P 274

Sonographische Überwachung des Follikelwachstums sowie der Ovulation bei normalen und stimulierten Zyklen

Funduk-Kurjak, B.; Kurjak, A 275

Vergleich zwischen Tamoxifen und Clomiphen zur Ovulationsinduktion

Messinis, I.E.; Nillius, S.J 277

Verhütung der Frühgeburtlichkeit bei Zwillingsschwangerschaften durch oral verabreichtes

Terbutalin

Skjaerris, J.; Aberg, A278

Der zyklische Wechsel der zervikalen Mikroflora und sein Effekt auf die Infektionen nach der Hysterektomie

Thadepalli, H.; Savage, E.W., Jr.; Salem, F.A.; Roy, I.; Davidson, E.C., Jr 280

Aktivität von «Natural Killer Cells» bei normaler Schwangerschaft sowie bei EPH-Gestose

Toder, V.; Blank, M.; Gleicher, N.; Voljovich, I.; Mashiah, S.; Nebel, L 281

VI

Inhaltsverzeichni

Beobachtung eines Organismus bei Patientinnen mit Trophoblasterkrankung und bei Patien tinnen mit EPH-Gestose

Lueck, J.; Brewer, J.I.; Aladjem, S.; Novotny, M 282

Die Rolle der Androgene bei Zyklusstörungen bei Frauen mit und ohne Hirsutismus und der Einfluss einer Glukokortikoidtherapie auf den Androgengehalt bei Frauen mit Hirsutis mus mit erhöhten androgenen Hormonwerten

Yuen, B.H.; Mincey, E.K 284

Akute Effekte des Rauchens auf die fetale Herzfrequenz

Lehtovirta, P.; Forss, M.; Kariniemi, V.; Rauramo, 1 
Mitteilung aus der Praxis

2 Fälle von Rektumperforation bei normaler Spontangeburt

Probst, H 289

Fortbildung

Einleitung

Gitsch, E 290

« Post-pill»- Amenorrhö

Tscherne, G 290

Extrakorporale Fertilisation

Winter, R 292

Die laparoskopische Tubensterilisation

Burmucic, R 293

Prostaglandine zur Abortinduktion und Geburtseinleitung

Lichtenegger, W 295

Autorenregister 297

Suppl. $1 \quad$ Tioconazole

A Review of Clinical Studies in Gynecology

Suppl. 2 Trichomoniasis

Scientific papers of the Symposium on Trichomoniasis Basel, October 20, 1983

Suppl. 3 Jahrestagung der Bayerischen Gesellschaft für Geburtshilfe und Frauenheilkunde und der Österreichischen Gesellschaft für Gynäkologie und Geburtshilfe

Juni 1983, Velden am Wörthersee

Suppl. 4 Schweizerische Gesellschaft für Gynäkologie und Geburtshilfe

Bericht über die Jahresversammlung, 23.-25. Juni 1983 in Lausanne

Société Suisse de Gynécologie et Obstétrique

Rapport de ГAssemblée annuelle, 23 au 25 juin à Lausanne

S. Karger · Medical and Scientific Publishers · Basel · München $\square$ Paris · London · New York · Sydney

Dosierungsangaben von Medikamenten

Autor und Herausgeber haben alle Anstrengungen unternom-men, um sicherzustellen, dass

Auswahl und Dosierungsangaben von Medikamenten im vorliegenden Text mit den ak-tuellen Vorschriften und der Praxis übereinstimmen. Trotzdem muss der Leser im Hinblick auf den Stand der Forschung, auf Änderungen staatlicher Gesetzgebungen und auf den ununterbrochenen Strom neuer Forschungsergebnisse bezüglich Medi-kamentenwirkung und Nebenwirkungen darauf aufmerksam gemacht werden, dass unbedingt bei jedem Medikament der Packungsprospekt konsultiert werden muss, um mögliche Änderungen hinsichtlich Indikation und Dosis nicht zu über-sehen. Gleiches gilt für spezielle Warnungen und Vorsichtsmassnahmen. Ganz besonders gilt dieser Hinweis für empfoh-lene neue und/oder nur selten gebrauchte Wirkstoffe.

Alle Rechte vorbehalten.

Ohne schriftliche Genehmigung des Verlags dürfen diese Publi-kation oder Teile daraus nicht in andere Sprachen übersetzt oder in irgendeiner Form mit mechanischen oder elektro-nischen Mitteln (einschliesslich Fotokopie, Tonaufnahme und Mikrokopie) reproduziert oder auf einem Datenträger oder einem Computersystem gespeichert werden.

S. Karger AG, Postfach, CH-4009 Basel (Schweiz) Printed in Switzerland by Thür AG Offsetdruck, Pratteln 